\title{
Investigation of the Influence of Microexplosions on a Laminar Premixed Water-in-Fuel Emulsion Spray Flame
}

\author{
N. Yokev ${ }^{1}$, J.B. Greenberg ${ }^{* 1}$ \\ ${ }^{1}$ Aerospace Engineering, Technion, Israel Institute of Technology, Haifa, Israel \\ *Corresponding author: aer9801@technion.ac.il
}

\begin{abstract}
A model is presented for a one-dimensional laminar premixed flame, propagating into a rich, off-stoichiometric, fresh homogenous mixture of water-in-fuel emulsion spray, with air and inert gas. The main purpose is to investigate the steady-state burning velocity and burnt temperature as functions of parameters such as initial water content in the emulsified droplet and total liquid droplet loading. In particular the influence of microexplosion of the spray's droplets on the flame's characteristics will be highlighted for the first time.
\end{abstract}

\section{Keywords}

water-in-fuel droplets; spray; microexplosions; laminar flame.

\section{Introduction}

It is common knowledge that tremendous effort is currently being invested in the search for viable biofuel blends for combustion engineering in an effort to reduce harmful NOx and PM emissions to the atmosphere. This effort has been spurred on by increasingly stringent international protocols governing the restricted emission of harmful pollutants to the atmosphere (see, for example, [1]). In practical terms, biofuel blends must be created to be fit for utilization in existing combustors (such as jet engines, for example) thereby circumventing the need to perform expensive modifications to current combustors or, more critically, to designing new suitable combustors.

Of the many ideas being considered to attempt to meet the strict standards dictated by protocols is the use of water-in-fuel emulsions. Consideration of water-in-fuel emulsion (hereinafter referred to as WIFE) droplets for combustion involves understanding a number of aspects of the physical behavior of these droplets as they evaporate, in terms of processes occurring within the droplet and their impact on the immediate vicinity outside the droplet. As will be described it is these processes that are responsible for the benefits that the droplets potentially possess, which may be advantageously harnessed under appropriate operating conditions.

Kadota and Yamasaki [2] appear to be the first authors to present a review dedicated to theoretical and experimental research on water fuel emulsion combustion until the year 2002. Subsequently, in 2014, Khan et al. [3] reviewed current trends in water-in-diesel emulsion as a fuel. Their paper focuses exclusively on experimental studies that were carried out to investigate the physics of both NOx and particulate reduction and the phenomenon of micro-explosion that may occur under appropriate operating conditions in WIFE (see later). In addition, there is a discussion of research into the actual influence of water-in-diesel emulsions on engine performance for a wide range of engine types and loading conditions. It is of interest to note that, due to the variety of engine set-ups and experimental methodologies employed, results reported in the literature were not consistent. However, generally, a water content of between $5-40 \%$ by volume was successfully utilized. Although there was a consensus concerning the ability of the water-in-fuel emulsions to reduce NOx and particulate production the reported extent of the reduction was far from uniform.

The first models of emulsified water/oil droplets appeared in 1977 [4, 5]. Jacques [4] solved numerically a heat transfer model containing essential elements of the underlying behavior of the transient heating of a single emulsified fuel droplet. His main concern was to try to quantify the primary effect of the water in the droplet. Attention was particularly focused on attempting to replicate the experimentally observed reduction of particulate formation by the water's presence based on the premise suggested by Shyu et al. [6] that, for waterless fuel droplets, this occurs during latter stages of heavy fuel oil combustion as a result of cracking of liquid phase hydrocarbons within the droplet at temperatures above $700 \mathrm{~K}$. The addition of water was shown to act as an extra source for heat loss thereby lowering the temperature within the droplet so that the extent of cracking reactions (and, therefore, subsequent formation of particulates) was reduced.

Micro-explosions seem to have been first reported in 1965 [7]. Experimental studies showed that combustion of compound droplets with different volatile fluids may be accompanied by violent explosion of the droplets. As the temperature increases, the higher volatile fluid, which is strategically located at the core, will evaporate sooner in the form of a bubble. The fast expansion of the gas at the core will result in partial or full disintegration of the 
parent droplet and it is this behavior that is called a micro-explosion. A similar occurrence called puffing happens when the nucleation bubble ruptures the parent droplet along with fine steams of tiny droplets. This happens when the bubble expands very rapidly and tears through the host droplet at a specific location. These phenomena are very important to combustion because they provide a secondary mechanism of atomization by breaking down large droplets into a smaller, faster evaporating form. Since the fuel combusts in gaseous form only, microexplosions help more fuel to change phase from liquid to gas, thereby improving the efficiency of fuel consumption .

One of the most important factors promoting the occurrences of micro-explosions is the superheat limit. Superheated liquid is defined as a fluid at extremely high temperatures - well above the boiling point, and thus occurs mainly when the working fluid undergoes a significant increase in pressure (Fu et al [8]). Since pressure is the main mechanism for controlling the boiling point of the fluid, micro-explosions could have major application in diesel engines, where the pressure is already designed to be extremely high (Law $[5,9,10])$. This phenomenon has a greater effect on heavier components (Fu et al [8]), such as fuel versus water, which means that a fuel shell will have higher tolerance to evaporation than water and it will allow more pressure to build up inside the emulsion droplet, promoting stronger micro-explosions. In [10], Law and Wang experimented with micro-explosions under different pressures, and showed an agreement with their previous mathematical theory. They theorized that increasing the pressure will enhance the occurrences of micro-explosions, and demonstrated this it up to 5 atmospheres.

Shinjo et al [11] described the detailed physics behind micro-explosions and puffing using numerical simulations to solve several problems such as the dynamics of the surfaces between emulsion droplet's fluids. The process of puffing begins with the growth of a single bubble between the water and fuel surfaces until it bursts. The outcome of the puffing depends on the following three mechanisms: (1) oscillation of the emulsified water droplet, (2) thrust created by the jet of vapor when the water boils, and (3) inertia of the bubble when it bursts - or in other words, the smaller the water content within the droplet is, the more effective the burst will be at detaching the water from the oil shell. On the other hand, if the water sub-droplet is large, the force applied by water vapor ejected from the emulsion droplet will push the water sub-droplet further within the oil shell - causing an increase in the effectiveness of the secondary atomization, but also the breakup time period. Since effective micro-explosions can improve fuel efficiency, a discussion of the increased time period is warranted. Tarlet et al [12] experimentally found that increasing either the water content or the droplet radius would promote longer micro-explosion delays. This concurs with the theoretical work of Shinjo et al [11], with respect to the addition of water content. Tarlet et al [12] also found that shorter micro-explosions delays occur when the ambient temperature and the relative velocity are higher, as well as an increase in the liquid content. He validated these conclusions with 27 different experiments under different conditions that would either promote or suppress the onset of micro-explosions. Finally, we mention a very recent theoretical study by Girin [13] which provides a solid analytical/theoretical foundation to the understanding of micro-explosions, as well as predicting the resulting number and size of the smaller fuel droplets that are formed.

Due to the complexity involved in modeling WIFE spray combustion under conditions in which micro-explosions take place, all theoretical studies we are aware of have only been concerned with the sequential evaporation of the fuel outer "shell" followed by the evaporation of the water inner "core". Thus, in a series of papers Hsuan et al [14], Hsuan and Lin [15,16] and Hou et al [17] considered WIFE spray flames. The mathematical analysis presented by these authors related to both fuel rich and fuel lean flames and took account of the fact that the major effect of the water evaporation was as a heat sink due to the heat loss incurred as the water absorbed heat for evaporation. Actually, such heat loss is also present when the outer fuel layer evaporates but the latent heat of evaporation of water is typically much larger than that of hydrocarbon fuels. For example, for n-octane it is of the order of $300 \mathrm{~kJ} / \mathrm{kg}$ in comparison to $2254 \mathrm{~kJ} / \mathrm{kg}$ for water. This discrepancy can have a strong effect on the laminar flame speed, depending on the relative amount of water in the droplets and the location of onset of pure water evaporation. With the respect to the latter both possible scenarios were examined (i.e. pre- and post- flame front water evaporation) combined with the nature of the fuel evaporation (completely before the flame front or only partially so). The methodology used was based on Lin et al's [18] analysis of fuel spray combustion which made use of asymptotic methods for finding the flame structure and an expression for the burning velocity. Critical conditions for flame extinction and propagation were determined as a function of the initial water content in the droplets.

An allied study, motivated by the behavior of emulsion explosives made up of a mixture of a fuel and an ammonium nitrate-water solution, was conducted by Hughes et al. [19]. A one-dimensional theoretical model was solved numerically to examine combustion waves driven by competing exothermic and endothermic reactions in the presence of water evaporation. A rich variety of influences of the water content on the flame propagation and its stability were noted, mainly because of its impact on the temperature and thereby on the fraction of ammonium 
nitrate consumed by the competing exothermic and endothermic reactions. Interestingly, the water evaporation was treated as a first-order chemical reaction with a specially constructed rate coefficient. However, once again, micro-explosions were not accounted for.

In the current work we describe a preliminary model of the behavior of a laminar off-stoichiometric water-in-fuel emulsion spray flame. We present for the first time a simplified theory for the steady state propagation of such flames and incorporate the overall effect of micro-explosions in the model. The governing equations are solved analytically and a formula for the burning velocity of the flame is derived which highlights the influence of the relative amount of water to liquid fuel in the sprays droplets and the impact of droplet micro-explosions.

\section{The model and governing equations}

\section{Assumptions}

We consider a laminar one-dimensional premixed flame propagating into a fuel rich off-stoichiometric fresh homogeneous mixture of fuel vapor, water-in-fuel emulsion droplets, oxygen and an inert gas. The flame is taken to propagate from right to left. The droplets are viewed from a far-field vantage point, i.e. their average velocity is equal to that of their host environment. For qualitative purposes this approach has been demonstrated to be quite valid [20]. The temperature of the droplets is taken to be that of the surroundings until the fuel's boiling temperature is reached; essentially the droplets heat-up time is small compared to the characteristic time associated with their motion. The initial ratio of the amount of water to the amount of liquid fuel in the droplets is assumed to be constant irrespective of the size of the droplets. Droplet evaporation is assumed negligible until a prescribed reference temperature (such as the boiling temperature of the liquid fuel) is attained at which point the fuel begins to evaporate at a finite rate. In the meantime, there is a buildup of water vapor within the compound droplets as the liquid water evaporates until conditions are attained for which the water vapor erupts through the outer layer of evaporating fuel thereby shattering the liquid fuel into smaller fuel-only droplets which, of course, continue to evaporate.

The stoichiometry of the gas mixture that the flame front meets is taken to be fuel rich, so that the limiting reactant consumed by chemical reaction is oxygen. It is assumed that the various transport coefficients, such as thermal conductivity, diffusion coefficient, specific heat at constant pressure, latent heat of vaporization of the droplets etc., can be satisfactorily specified by representative constant values. An overall reaction of the form $v_{F}$ fuel $+v_{O}$ oxidant $\rightarrow$ products is taken to describe the chemistry. However, in this way the water vapor does not participate in the chemical reaction. Nevertheless, neutralizing the role of chemical effects associated with water vapor by utilizing a one-step chemical reaction is not unreasonable at a first attempt, as it was found [21] that evaporated water vapor has less than a $10 \%$ effect on flame extinction conditions whereas thermal effects, that are included in our model, influence them significantly

As the velocity of propagation of the flame is much less than the velocity of sound, dynamic compressibility effects in the mixture can be neglected. Thus, the density becomes only a function of the temperature through the gas law.

A realistic polydisperse spray can be described using the sectional method [22] in which the droplet sizedistribution is divided into sections (or bins) and conservation equations are derived for the liquid fuel in each section allowing for droplet evaporation from a given section, say $\mathrm{j}$, and addition to that section as droplets evaporate in the next section up and become eligible for membership in j. For simplicity at the current stage we assume a mono-sectional description of the spray's droplets.

Droplet evaporation is assumed negligible until a prescribed reference temperature $T_{v}$ (such as the boiling temperature of the liquid fuel) is attained. The pre-vaporization region will be denoted: $R_{1}=\left\{\xi:-\infty<\xi \leq-\xi_{v f}\right\}$ where $\xi=-\xi_{v f}$ is the point at which the ambient temperature equals $T_{v}$. At this point the liquid fuel in the droplets begins to evaporate. Micro-explosion is supposed to occur instantaneously at a predefined temperature $T_{e}$, producing smaller droplets which will evaporate more rapidly than the parent droplets from which they were created. This region will be denoted by $R_{2}=\left\{\xi:-\xi_{v f} \leq \xi \leq-\xi_{e}\right\}$. The stoichiometry of the gas mixture that the flame front meets will be determined by the particular conditions under consideration. We restrict our analysis to those circumstances in which a homogeneous flame front actually exists. If the flame front is located at $\xi=0$ in the moving coordinate system we adopt, the pre- and post-flame zones can be denoted by $R_{3}=\left\{\xi:-\xi_{e} \leq \xi \leq 0\right\}$ and $R_{4}=\{\xi: 0 \leq \xi<\infty\}$, respectively.

\section{Governing equations}

Under the aforementioned assumptions the governing equations for the gas-phase describe energy and oxygen mass fraction conservation: 


$$
\begin{aligned}
& T^{\prime}=T^{\prime \prime}+D a \cdot m_{O} \exp \left[\theta\left(1-T^{-1}\right)\right]-L_{W} S_{v W}-L_{f} S_{v f} \\
& m_{O}^{\prime}=m_{O}^{\prime \prime}-D a \cdot m_{O} \exp \left[\theta\left(1-T^{-1}\right)\right] \\
& m_{d f}^{\prime}=-S_{v f} \\
& m_{d W}^{\prime}=-S_{v W}
\end{aligned}
$$

where ( $)^{\prime}$ denotes the derivative with respect to $\xi$, the normalized mass fractions of oxygen, liquid fuel and water are defined by

$m_{O}=\frac{m_{O}^{*}}{m_{O u}^{*}}, m_{d f}=\frac{m_{d f}^{*}}{m_{f u}^{*}+\left(m_{d f u}^{*}+m_{d W u}^{*}\right)}, m_{d W}=\frac{m_{d W}^{*}}{m_{f u}^{*}+\left(m_{d f u}^{*}+m_{d W u}^{*}\right)}$

with the upper asterisk denoting actual mass fraction and the suffixes $O, u, f, d$ and $W$ denoting oxygen, unburned value, fuel, droplets and water, respectively. $T$ is the non-dimensional temperature, $D a$ is the usual chemical Damkohler number, $\theta$ is the dimensionless activation energy, $L_{W}, L_{f}$ are the latent heats of vaporization of the water and fuel, respectively, and where the evaporation source terms for fuel and water are

$$
S_{v f}=S_{f}\left(H\left(\xi+\xi_{v f}\right)\left(1-H\left(\xi_{e}+\xi\right)\right)\right)+f S_{f} H\left(\xi+\xi_{e}\right), \quad S_{v W}=S_{W} H\left(\xi+\xi_{e}\right)
$$

in which, following the sectional approach to spray modelling [22]:

$$
S_{f}=C_{f} m_{d f}, S_{W}=C_{W} m_{d W}
$$

where $C_{f}$ and $C_{W}$ are evaporation Damkohler numbers. In Eqs.(6a) and (6b) use is made of the Heaviside function $H$ to define the regions in which the expressions it multiplies apply. Thus, in Eq.(6a) the first term on the right hand side is applicable once the fuel starts to evaporate through to the point $\xi=-\xi_{e}$ where the microexplosion occurs. From that point onwards the rate of evaporation of the fuel is increased by a factor of $f$ due to the shattering of the droplets by the micro-explosion. Incorporating a result of Girin's micro-explosion theory [13] within the framework of the sectional approach to spray modelling, an expression for this factor can be shown to be

$$
f \approx\left(0.27\left(1-\alpha^{1 / 3}\right)\right)^{-2}
$$

where $\alpha$ is the initial ratio of water to liquid fuel in the spray's droplets. As mentioned previously this ratio is assumed to be the same for all the droplets initially in the spray. In what may be considered as the current first order model the sudden micro-explosion at $\xi=-\xi_{e}$ simply results in the same amount of remaining fuel instantaneously changing its rate of evaporation. Other features of the occurrence of a micro-explosion, such as complete droplet rupturing puffing [11] and the spatial location of the new sized droplets, are not accounted for at the current stage. The evaporation of the water is assumed to occur within the original compound droplets and the micro-explosion releases all water vapor instantaneously at $\xi=-\xi_{e}$ so that $C_{W} \rightarrow \infty$. This fact will express itself via the matching conditions between regions $R_{2}$ and $R_{3}$.

To close this mathematical formulation boundary and matching conditions are required. These are readily shown to be

$$
\begin{aligned}
& \xi \rightarrow-\infty \quad T=T_{u}, m_{O}=1, m_{d f}=m_{d f u}, m_{d W}=m_{d W u} \\
& \xi \rightarrow \infty \quad T=T_{b}, m_{O}=0 \\
& \xi=-\xi_{v f} \quad T=T_{v},\left[m_{O}\right]=\left[m_{O}^{\prime}\right]=\left[T^{\prime}\right]=0, m_{d f}=m_{d f u} \\
& \xi=-\xi_{e} \quad T=T_{e},\left[m_{o}\right]=\left[m_{o}^{\prime}\right]=[T]=\left[m_{d f}\right]=0,\left[T^{\prime}\right]=L_{W} m_{d W u} \\
& \xi=0 \quad[T]=\left[m_{O}\right]=\left[m_{d f u}\right]=0,\left[T^{\prime}\right]=-Q,\left[m_{O}^{\prime}\right]=Q
\end{aligned}
$$

where the subscript $u$ denotes unburned conditions.

\section{Solution}

The solution to the afore-defined problem is readily developed using known asymptotic methods, and is found to be

In $R_{1}$ :

$T(\xi)=\left[1-L_{w} \alpha \tilde{\delta} e^{\xi_{e}}-\frac{C_{f} f L_{f}[(1-\alpha) \widetilde{\delta}] e^{-C_{f}\left(-\xi_{e}+\xi_{v f}\right)+\xi_{e}}}{C_{f} f+1}-\frac{C_{f} L_{f}[(1-\alpha) \widetilde{\delta}] e^{\xi_{v f}}}{C_{f}+1}+\frac{C_{f} L_{f}[(1-\alpha) \widetilde{\delta}] e^{-C_{f}\left(-\xi_{e}+\xi_{v f}\right)+\xi_{e}}}{C_{f}+1}\right] e^{\xi}$

$m_{0}=1-e^{L e \xi}$ 
$m_{d f}=(1-\alpha) \tilde{\delta}$

In $R_{2}$ :

$T(\xi)=$

$-L_{f}[(1-\alpha) \tilde{\delta}]+\left(1-L_{w} \alpha \tilde{\delta} e^{\xi_{e}}-\frac{C_{f} f L_{f}[(1-\alpha) \tilde{\delta}] e^{-C_{f}\left(-\xi_{e}+\xi_{v f}\right)+\xi_{e}}}{C_{f} f+1}+\frac{C_{f} L_{f}[(1-\alpha) \tilde{\delta}] e^{-C_{f}\left(-\xi_{e}+\xi_{v f}\right)+\xi_{e}}}{C_{f}+1}\right) e^{\xi}+\frac{L_{f}[(1-\alpha) \tilde{\delta}] e^{-C_{f}\left(\xi+\xi_{v f f}\right)}}{C_{f}+1}(15 a)$

$m_{0}=1-e^{L e \xi}$

$m_{d f}=(1-\alpha) \tilde{\delta} e^{-C_{f}\left(\xi+\xi_{v f}\right)}$

In $R_{3}$ :

$T(\xi)=-L_{w} \alpha \tilde{\delta}-L_{f}[(1-\alpha) \tilde{\delta}]+e^{\xi}+\frac{L_{f}[(1-\alpha) \widetilde{\delta}] e^{-C_{f}\left[\left(-\xi_{e}+\xi_{v f}\right)+f\left(\xi+\xi_{e}\right)\right]}}{C_{f} f+1}$

$m_{0}=1-e^{L e \xi}$

$m_{d f}=(1-\alpha) \tilde{\delta} e^{-C_{f}\left[\left(-\xi_{e}+\xi_{r f}\right)+f\left(\xi+\xi_{e}\right)\right]}$

In $R_{4}$ :

$T(\xi)=1-L_{w} \alpha \tilde{\delta}-L_{f}[(1-\alpha) \tilde{\delta}]+\frac{L_{f}[(1-\alpha) \tilde{\delta}] e^{-C_{f}\left[\left(-\xi_{e}+\xi_{v f}\right)+f\left(\xi+\xi_{e}\right)\right]}}{C_{f} f+1}$

$m_{O}=0$

$m_{d f}=(1-\alpha) \tilde{\delta} e^{-C_{f}\left[\left(-\xi_{e}+\xi_{v f}\right)+f\left(\xi+\xi_{e}\right)\right]}$

The locations of the onset of evaporation and the micro-explosion are determined according to predefined temperatures and can be readily found to be:

$$
\begin{aligned}
& -\xi_{v_{f}}=\ln \left\{\frac{T_{v f}+\frac{C_{f} L_{f}[(1-\alpha) \tilde{\delta}]}{C_{f}+1}}{1-L_{w} \alpha \widetilde{\delta} \xi_{e}-\frac{C_{f} f L_{f}[(1-\alpha) \tilde{\delta}] e^{-C_{f}\left(-\xi_{e}+\xi_{v f}\right)+\xi_{e}}}{C_{f} f+1}+\frac{C_{f} L_{f}[(1-\alpha) \tilde{\delta}] e^{-C_{f}\left(-\xi_{e}+\xi_{v f}\right)+\xi_{e}}}{C_{f}+1}}\right\} \\
& -\xi_{e}=\ln \left\{T_{e}+L_{f}[(1-\alpha) \tilde{\delta}]+L_{w} \alpha \tilde{\delta}-\frac{L_{f}[(1-\alpha) \tilde{\delta}] e^{-C_{f}\left(-\xi_{e}+\xi_{v f}\right)}}{C_{f} f+1}\right\}
\end{aligned}
$$

The burning velocity is determined to be:

$U=\frac{R T_{a} \sqrt{2 L e \lambda A}}{E} * \exp \left\{\frac{-E}{2 R T_{a}^{*}}-\frac{L_{f^{\prime}}[(1-\alpha) \widetilde{\delta}]}{2}\left[1-\frac{U^{2} e^{-\frac{C_{f}^{*} \lambda\left[\left(-\xi_{e}+\xi_{v f}\right)+f \xi_{e}\right]}{U^{2}}}}{C_{f}{ }^{*} \lambda \cdot f+U^{2}}\right]-\frac{L_{w^{\prime} \alpha \widetilde{\delta}}}{2}\right\}$

in which $T_{a}$ is the adiabatic flame temperature, $\lambda$ is the thermal conductivity of the gas mixture, $R$ is the gas constant, $E, A$ are the activation energy and pre-exponential coefficient of the global chemical reaction and $\left(L_{f}^{\prime}, L_{W}^{\prime}\right)=\theta\left(L_{f}, L_{W}\right)$ are related to the latent heats of evaporation of the fuel and water, respectively.

Note that since $C_{f}$ is in non-dimensional form according to $C_{f}=C_{f}^{*} \lambda / U^{2}$ Eq.(20) becomes an implicit equation for the burning velocity. In the aforementioned solutions the total initial fraction of liquid in the total fuel and water (vapor +liquid) in the fresh mixture, $\tilde{\delta}$, appears. It can be shown that if there are no droplets present initially (i.e. $\tilde{\delta}=0)$ and the premixture is purely gaseous the expression for the flame propagation velocity collapses to that for a gaseous flame (see, e.g. Buckmaster and Ludford [23]). If there is no water present in the droplets (i.e. $\alpha=0$ ) the formula reduces to that of Greenberg et al. [24] in the limit of infinite and finite vaporization rate. The second and third terms in the exponential represent the contributions to reducing the burning velocity (relative to that of a purely gaseous flame) due to evaporation of the liquid fuel and the water, respectively. 


\section{Results and discussion}

By specifying the initial fraction of liquid in the total fuel and water (vapor +liquid) in the fresh mixture, $\tilde{\delta}$ and the initial ratio of water to liquid in the droplets, $\alpha$, it can be shown that the mass fractions in the fresh mixture are given by the following expressions:

$$
\begin{aligned}
& m_{O u}^{*}=s\left\{1-m_{d f u}^{*}-m_{f u}^{*}-m_{d W u}^{*}\right\} \\
& m_{d f u}^{*}=\frac{\frac{\delta \delta \varphi}{\hat{\alpha}_{o f}}}{\left[\left(1+\frac{s \varphi}{(1-\alpha) \hat{\alpha}_{o f}}\right)+\frac{\alpha(1-\tilde{\delta})}{(1-\alpha)}\right]} \\
& m_{d W u}^{*}=\frac{\alpha m_{d f u}^{*}}{(1-\alpha)} \\
& m_{f u}^{*}=1-\frac{m_{o u}^{*}}{s}-m_{d f u}^{*}-m_{d w u}^{*}
\end{aligned}
$$

where $S$ is the mole fraction of oxygen in the fresh mixture, $\alpha_{O F}$ is the stoichiometric coefficient and $\varphi$ is the equivalence ratio. Here, $\varphi$ is taken as 2 and $\alpha_{O F}=3.5$.

Other data used was as follows (unless otherwise specified):

$q=1.279 \cdot 10^{7}\left[\frac{\mathrm{J}}{\mathrm{kg}}\right], \rho=1\left[\frac{\mathrm{kg}}{\mathrm{m}^{3}}\right], \kappa=0.02512\left[\frac{\mathrm{W}}{\mathrm{mK}}\right], C_{p}=1255.92 \frac{\mathrm{J}}{\mathrm{kg}}, A=10^{10}\left[\frac{1}{\mathrm{sec}}\right], E=2 \cdot 10^{8}\left[\frac{\mathrm{J}}{\mathrm{kmol}}\right], T_{u}^{*}=$ $300[K], T_{v f}^{*}=400[K], T_{e}^{*}=600 K, L_{f} / q=0.04, L_{W} / q=0.12$.

In Figure 1 we illustrate the major effect the micro-explosion has on the burning velocity. The burning velocity is plotted against the total initial liquid mass fraction (i.e. water+liquid fuel). Three different initial water content fractions are considered. Curves are drawn accounting for the occurrence of micro-explosions (the solid lines) and for the cases when no micro-explosion is accounted for (the broken lines) for comparison. The impact of the microexplosion is very pronounced, reducing the burning velocity by a factor of almost $43 \%$ at the most, when no fuel vapor is initially present in the fresh mixture. This factor decreases as less liquid is replaced by pre-vaporized fuel. In addition, it is seen that the more water initially present in the spray's droplets the greater the impact in reducing the burning velocity. This is evidently due to the greater heat absorbed by the water for the sudden evaporation at the point of the micro-explosion which, in turn, reduces the temperature and thereby the propagation velocity. This effect is likely play a critical role in the stability (and extinction of these flames). The combined effect of volumetric heat loss and liquid fuel evaporation heat loss on flame extinction was addressed in [25] and compared to the case of volumetric heat loss alone [26]. However, an analysis of these features in the current context is beyond the scope of the current paper.

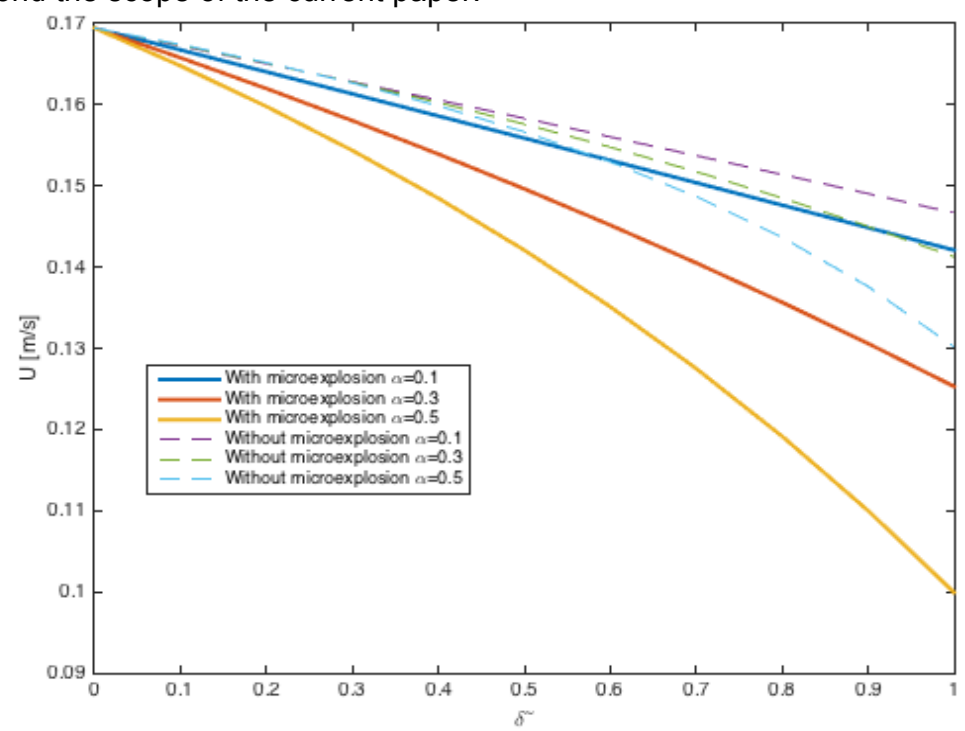

Figure 1: Influence of droplets micro-explosion on the burning velocity of water-in-fuel laminar spray flames as a function of total initial liquid mass fraction.

In Figure 2 the influence of the micro-explosion parameter $f$ on the flame temperature is illustrated for different initial liquid mass fractions. Recall that the factor $f$ depends on the initial fraction of water in the droplets (Eq.(8)). It is important to note that the flame temperature depends amongst others on both droplet loading and the water content. Thus, when there are no droplets present the top portion of the graph is unchanged with no heat loss accrued. As liquid droplets are introduced the effect that the water content $\alpha$ has on the temperature comes to the 
fore. Since $f$ is a function of alpha, the more water in the droplets the larger $f$ becomes with the consequent microexplosion producing smaller droplets which evaporate more rapidly thereby focusing heat loss due to the absorption of heat by the rapidly evaporating fuel droplets. This yields lower temperatures.

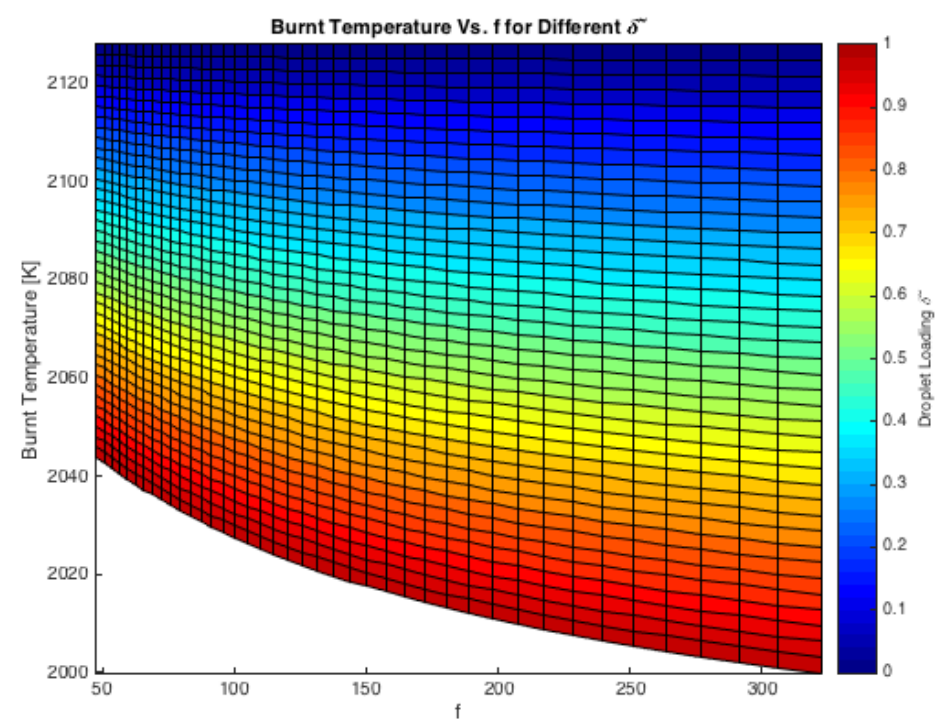

Figure 2: Influence of micro-explosion factor $f$ on flame temperature in water-in-fuel spray flame propagation.

In Figure 3 the effect of the micro-explosions on the behavior of the liquid fuel mass fraction is drawn. The upper curve is for the case when no water is present in the sprays droplets so that they are comprised of liquid fuel only. For the data considered here this spray evaporates fairly slowly and droplets actually traverse the flame front, which is located at $\xi=0$. The lower curve illustrates the behavior when micro-explosions occur at about $\xi=-2.2$

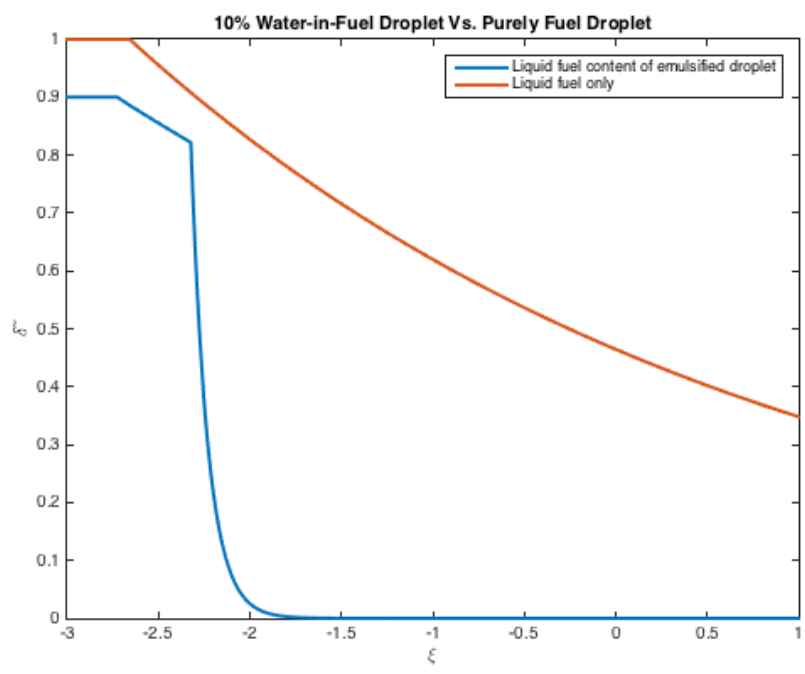

Figure 3: Comparison between liquid fuel spatial profiles with and without accounting for droplet micro-explosions.

The influence of the micro-explosions is dramatic, with the smaller droplets produced evaporating very quickly. In contrast to the purely liquid fuel spray, the water-in-fuel spray considered here are completely evaporated upstream of the flame front. Nevertheless, as shown above, the price paid for the premature fuel evaporation is a drastic loss in the flame temperature which may, of course, be detrimental in terms of combustion efficiency. It would seem that micro-explosions are likely to be more efficient in near stoichiometric or fuel lean mixtures for which the prematurely produced fuel vapor is actively involved in the combustion at the flame front. Such a 
situation is precluded here as the mixture is off-stoichiometric and fuel rich so that the limiting reactant accounted for in the global chemical reaction assumed is the oxidant.

\section{Conclusions}

A simple model of water-in-fuel laminar spray flame propagation was presented for the first time, including the influence of micro-explosions. The analytical solutions provide insight into the essential processes competing in such combustion. For the fuel-rich off-stoichiometric flames considered it was found that the micro-explosions are effective in enhancing the rapid production of fuel vapour but are rather disadvantageous in terms of lowering the flame temperature and burning velocity. The latter effects are likely to impinge directly on the stability and/or extinction of such flames - an issue that is the subject of ongoing research. The primary advantage of microexplosions is likely to be prominent in lean and near-stoichiometric mixtures. Work in this direction will be reported on in the future.

\section{Acknowledgements}

JBG thanks the Lady Davis Chair in Aerospace Engineering for partial support of this research.

\section{References}

[1] The Kyoto Protocol Mechanisms, International Emissions Trading Clean Development Mechanism Joint Implementation, United Nations Framework Convention on Climate Change, 2007.

[2] Kadota, T. and Yamasaki, H. , 2002, Progress in Energy and Combustion Science, 28, pp.385-404.

[3]. Khan, M.Y., Karim, Z.A.A, Hagos, F.Y., Aziz, A.R.A and Tan, I.M., 2014, The Scientific World Journal, Article ID 527472.

[4] Jacques, M.T., 1977, Combustion and Flame, 29, pp. 77-85.

[5] Law, C.K.,1977, Combustion Science and Technology,17, pp. 29-38.

[6] Shyu, R.R., Chen, C.S., Goudie, G.O. and El-Wakil, M.M., 1972, Fuel, 51, pp. 135-145.

[7] Ivanov, V.M and Nefdov, P.I., 1965, NASA TT F-258.

[8] Fu, W.B., Hou, L.Y., Ma, F.H. and Wang, L., 2002, Fuel Processing Technology, 79, pp. 107-119.

[9] Law, C.K., Lee, C.H. and Srinivasan, N., 1980, Combustion and Flame, 37, pp. 125-143.

[10] Law, C.K and Wang, C.H., 1985, Combustion and Flame, 59, pp. 53-62.

[11] Shinjo, J., Xia, J., Ganippa, L.C. and Megaritis, A., 2014, Physics of Fluids, 26, pp. 103302, 1-22.

[12] Tarlet, D., Bellettre, J.,Tazerout, M. and Rahmouni, C., 2009, International Journal of Thermal Science, 48, pp. 449-460.

[13] Girin, O.G., 2016, ILASS-Europe 2016, 27th Annual Conference on Liquid Atomization and Spray Systems, 4-7 September, Brighton, UK.

[14] Hsuan, C.-Y., Hou, S.-S and Lin, T.-H., 2014,, Mathematical Problems in Engineering, Article ID 584926.

[15] Hsuan, C.-Y. and Lin, T.-H, 2012, Combustion and Flame,159, pp. 1652-1662.

[16] Hsuan, C.-Y. and Lin, T.-H, 2012, Combustion Theory and Modeling,16(3), pp.483-505.

[17] Hou, S.-S., Lin, J.-C. and Hsuan, C.-Y., 2014, International Journal of Heat and Mass Transfer, 71, pp. 503514 .

[18] Lin, T.H., Law, C.K. and Chung, S.H., 1988, International Journal of Heat and Mass Transfer, 31(5), pp. 1023-1034.

[19] Hughes, K.J., Brindley, J. and McIntosh, A.C., 2013, Proceedings of the Royal Society A, 469: 20130506, http://dx.doi.org/10.1098/rspa.2013.0506.

[20] Greenberg, J.B. and. Kalma, A., 1998, "Computational Aspects of the Sectional Modeling Method for Predicting Spray Combustion", HTD-Vol. 361-2, Proceedings of the ASME Heat Transfer Division, pp. 3-10, ASME International Mechanical Engineering Congress, Anaheim, Ca.

[21] Lentati, A.M. and Chelliah, H.K., 1998, Proceedings of the Combust Institute,.27, pp. 2839-46.

[22] Greenberg, J.B., Silverman, I. and. Tambour, Y., 1993, Combustion and. Flame,. 93, pp. 90-963.

[23] Buckmaster, J.D. and Ludford, G.S.S., Theory of Laminar Flames, Cambridge University Press, Cambridge, UK, 1982.

[24] Greenberg,J.B.,McIntosh, A.C. and Brindley, J.,2001, Proceedings of the Royal Society of London, Series A: Mathematical, Physical and Engineering Sciences, 457, No.2005, pp.1-31.

[25] Kats, G. and Greenberg, J.B., 2016, Journal of Combustion, Article ID 1069873, Vol. 2016, http://dx.doi.org/10.1155/2016/1069873.

[26] Joulin, G. and Clavin, P., 1979, Combustion and Flame, 35, pp. 139-153. 